\title{
13. Epidural blood patch in the management of the syndrome of spontaneous intracranial hypotension: An effective therapeutic approach
}

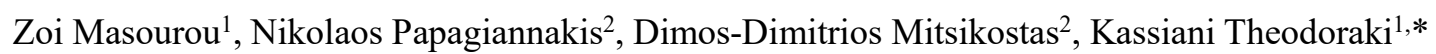

${ }^{1}$ A' Anaesthesiology Clinic, Pain Relief and Palliative Care Centre, Aretaieion University Hospital, National and Kapodistrian University of Athens, Athens, Greece; ${ }^{2}$ Neurology Department, Aiginiteion University Hospital, National and Kapodistrian University of Athens, Athens, Greece. * ktheodoraki@hotmail.com

Background: Spontaneous intracranial hypotension $(\mathrm{SIH})$ is a rare syndrome characterized by heterogeneity of presentation and prognosis, which can occasionally result in serious complications, such as the formation of subdural hematoma (SDH). This case series aims to emphasize that SIH remains a diagnostic and therapeutic challenge; it can present with a far broad clinical spectrum of symptoms, can lead to SDH and if conservative treatment fails, an epidural blood patch (EBP) is a viable treatment option. Although the exact aetiology of SIH is not known, it is believed to be due to cerebrospinal fluid (CBF) leak or a low CBF pressure.

Case report: Three patients (two males and one female) with age ranging between 38-53 years old who presented with complaints not only of an orthostatic headache, but with a variety of symptoms of SIH, including the formation of two SDHs in one of them, were included in this series. These patients did not respond to conservative management and subsequently, given the clinical and radiological evidence of SIH, were referred to the Anaesthesia Department for an EBP. The exact site of the CSF leak was identified with imaging modalities, including magnetic resonance imaging (MRI) of the brain and spinal cord, prior to the EBP. All three patients were subjected to an EBP with an 18-gauge epidural needle placed into the middle epidural compartment at the T12-L3 level. A total of between $30-43 \mathrm{~mL}$ of autologous blood was collected from the patients' left basilic vein and was injected into the epidural space under strict aseptic conditions. Two lumbar (L1-L2, L2-L3) and one thoracic (T11-T12) EBPs were performed on the three patients. All patients reported complete resolution of symptoms following the EBPs, while MRI imaging improved substantially.

Discussion: This report describes three cases of SIH with CSF leak originating from the cervical, the thoracic and the lumbar level. The EBP restored CSF volume and relieved the patients' persistent symptoms. MRI helps in showing indirect signs of low volume of CSF, though it may not be possible to find the actual site of leak. In conclusion, EBP is a wellaccepted and beneficial treatment modality for SIH when conventional measures fail.

\section{References}

[1] Limaye K, Samant R, Lee RW. Spontaneous intracranial hypotension: diagnosis to management. Acta Neurologica Belgica. 2016; 116: 119-125.

[2] Mokri B. Spontaneous low pressure, low CSF volume headaches: spontaneous CSF leaks. Headache. 2013; 53: $1034-1053$. 